\title{
Formación de habilidades de investigación en estudiantes de ingeniería como parte del Servicio Social
}

Danice Deyanira Cano Barrón ${ }^{1}$ y Humberto José Centurión Cardeña ${ }^{1}$

\section{Resumen}

La necesidad actual de desarrollar tecnología en las universidades es un reto que poco a poco ha empezado a encararse siguiendo diversas estrategias, la experiencia que aquí se comparte es un mecanismo diseñado para responder de manera conjunta a dos elementos distintivos: desarrollar en los estudiantes el gusto por la investigación y fortalecer las actividades del programa institucional de Servicio Social. Se describe el proceso de elaboración de una propuesta de desarrollo tecnológico para un congreso por parte de los estudiantes, así como los resultados obtenidos y la evaluación por parte de los participantes. En términos generales los estudiantes encontraron la experiencia innovadora y gratificante, que aporta un elemento diferenciador a su perfil profesional y los motiva a continuar desarrollando este tipo de actividades.

Palabras Clave: Investigación, Servicio Social, Desarrollo Tecnológico, Educación Superior, Habilidades profesionales.

Fecha de recepción: julio 2013 | Fecha de aceptación: octubre 2013

${ }^{1}$ Instituto Tecnológico Superior de Motul, Depto. de Ingeniería en Sistemas Computacionales, México. 


\section{Abstract}

The current need to develop technology in universities is a challenge that has slowly started to be addressed following different strategies. The experience shared here is a mechanism designed to jointly respond to two distinct elements: encourage students to research and strengthen the activities of the Social Service institutional program. It describes the process of technological development for a conference by students, as well as the results obtained and evaluation by the participants. Generally, students found the experience as innovative and rewarding, providing a differentiator to their professional profile and encouraging them to continue developing these activities.

Keywords: Research, Social Service, Technological Development, Higher Education, Professional Skills. 
La sociedad actual demanda de las universidades no solo servicios educativos y culturales, sino también la producción de conocimiento nuevo que satisfaga las necesidades de gestión y transferencia tecnológica que contribuyan a la solución de los problemas de la sociedad (Monroy, 2007). Educación, producción y transferencia tecnológica, progreso de la sociedad son conceptos que debidamente alineados y utilizados de manera efectiva son factores que impactan directamente en el desarrollo económico de cualquier país. La educación enfocada a la producción de tecnología permite generar nuevos descubrimientos aplicados a áreas sentidas como pertinentes resultando en la mejora de las condiciones de vida de las personas y eventualmente si esa tecnología se patenta impacta en el crecimiento de un país.

Al momento actual, las universidades están más que conscientes de la nueva demanda que se hace de ellas y aunque algunas ya están atendiendo las tareas que la sociedad les ha encomendado cambiando su enfoque puramente científico a un enfoque de tecnología aplicada a las necesidades del entorno, son necesarios mayores esfuerzos financieros del gobierno y de los particulares que apoyen la investigación científica y la transferencia tecnológica

En México, en los últimos 10 años las principales universidades públicas sólo han generado alrededor de 80 patentes que benefician directamente a la sociedad. Durante ese periodo la UNAM patentó 43 proyectos; la Universidad Autónoma Metropolitana, 23, y el Instituto Politécnico Nacional 12 (Olivares, 2007). Si se considera que éstas son las Instituciones de mayor antigüedad y desarrollo del país, con el mejor equipamiento y la mayor capacidad de producción de tecnología, los productos no son los que se esperarían y demuestran el poco interés en generar tecnología vanguardista. Y es que si se analiza bien, UNAM ha generado poco más de 4 patentes al año, mientras que las dos Universidades restantes sólo han producido 2 y 1, respectivamente, esto habla de la necesidad de transformar la visión de los científicos a capitalizar sus investigaciones en patentes que reditúen y mejoren la vida de sus compatriotas.

En este mismo sentido se ha observado que de los 4.5 millones de empresas que hay en el país, sólo 20 han generado patentes (Olivares, 2007), quedando de manifiesto que la visión empresarial se enfoca en el consumo de la tecnología más que en la producción de ella. Por lo que analizando las dos principales fuentes potenciales de patentes y por consecuencia de tecnología, se observa que México tiene la necesidad de poner en marcha programas y estímulos que motiven, favorezcan y fortalezcan la producción de propiedad intelectual ayudando a la vinculación empresa - universidades y a la producción de avances que mejoren los productos y servicios que se ofertan mejorando e innovando la tecnología que subyace en ellos.

Es pertinente destacar que existen indicios que este fenómeno es de tipo cultural, ya que hay evidencia del trabajo de las universidades relacionadas con múltiples creaciones e innovaciones tecnológicas y científicas, pero poco interés por patentar esos 
productos (Loera \& Carrillo, 2004), por lo que una buena estrategia para incrementar estos indicadores consistiría en concientizar a los investigadores de la necesidad de generar dichos registros y las ganancias asociadas, ya que la dinámica de parear el trabajo de investigación formal con las necesidades percibidas de las industrias, marcan las pautas a seguir con sus necesidades y la forma de resolverlas dan lugar a una innovación tecnológica que puede resultar en un nuevo producto o servicio.

\section{Instituto Tecnológico Superior de Motul (ITS Motul) y el programa de Servicio Social}

El ITS Motul es un organismo público descentralizado del Gobierno del Estado de Yucatán, México; cuya creación responde a la necesidad de formar jóvenes en las poblaciones mayas circunvecinas a la ciudad de la que toma el nombre. El Instituto tiene como finalidad, formar profesionales para sostener la planta productiva del país, así como impulsar y desarrollar modelos de aplicación relativos a las ingenierías y tecnologías en las áreas agropecuarias, industrial y de servicios. Es una institución de educación superior relativamente joven con apenas 11 años de creación y en vías de desarrollo.

Actualmente cuenta con 672 alumnos y 39 catedráticos, distribuidos en cinco carreras de Ingeniería: Industrial, Electromecánica, Sistemas Computacionales, Electrónica y Energías Renovables, esta última de reciente creación.

Debido a que la Institución, sus profesores y administración son aún muy jóvenes, las actividades de profesores y estudiantes se orientan todavía en gran parte a la formación y desarrollo de habilidades profesionales. Sin embargo, siguiendo las políticas Estatales y Nacionales se considera como pertinente la generación de conocimiento tecnológico aplicado, tanto de parte de los profesores pero más aún se busca empezar a involucrar a los estudiantes en actividades de investigación que les permita vincularse de manera más completa y propositiva al sector productivo y promover esta habilidad como una actitud sello de los egresados.

En términos generales se entiende por Servicio Social el trabajo de carácter temporal y obligatorio, que institucionalmente presten y ejecuten los estudiantes en beneficio de la sociedad (Dirección General de Educación Superior Tecnológica, 2013), y que todos los estudiantes requieren realizar por al menos 480 horas o 6 meses (lo que ocurra primero) en alguna dependencia de gobierno para cubrir con este requisito de titulación y egreso.

En particular el Servicio Social dentro del sistema tecnológico tiene por objeto: convertir esta prestación en un acto de reciprocidad para con la sociedad a través de los planes y programas del sector público y contribuir a la formación académica y capacitación profesional del prestador del Servicio Social (Dirección General de Educación Superior Tecnológica, 2013). Se fortaleció de manera directa y simultánea la formación académica y profesional de los estudiantes a través del 
desarrollo de proyectos que requieran del uso de los conocimientos y habilidades en actividades relacionadas con el desarrollo de aplicaciones que pudieran ser consideradas como innovadoras y ser presentadas en algún congreso especializado.

Generalmente los estudiantes realizan sus actividades en diversos departamentos de dependencias gubernamentales (escuelas, ayuntamientos, bibliotecas públicas, etc.) en su mayoría realizando actividades administrativas poco relacionadas con el desarrollo de su perfil profesional, por lo que dichas asignaciones cumplen con la mitad del enfoque del servicio social ya que aunque sí cubren con la parte de apoyar el desarrollo de planes y programas del sector público no se percibe el desarrollo de habilidades relacionadas con el perfil profesional.

\section{Proyecto de Servicio Social e Investigación}

Se decidió trabajar de manera multidisciplinaria incluyendo a estudiantes de la carrera de Ingeniería en Sistemas y de Electromecánica, trabajando alrededor de dos actividades substanciales, la primera era el Concurso Nacional de Robótica del Sureste como medio para desarrollar habilidades de programación tanto desde el punto de vista de los sistemas computacionales como desde la robótica y el segundo un proyecto de desarrollo de una aplicación relacionado directamente con su carrera y que sería diseñado y delimitado en conjunto por profesor y estudiantes.
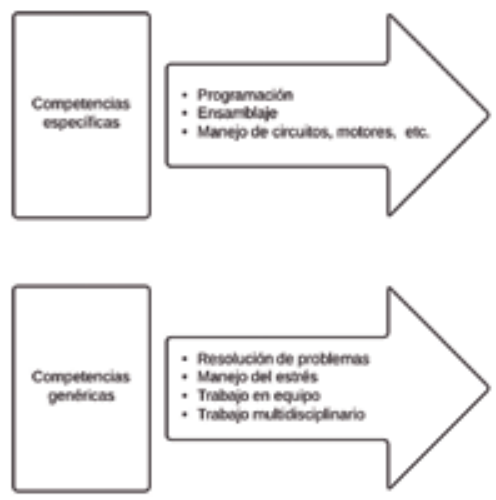

(A)
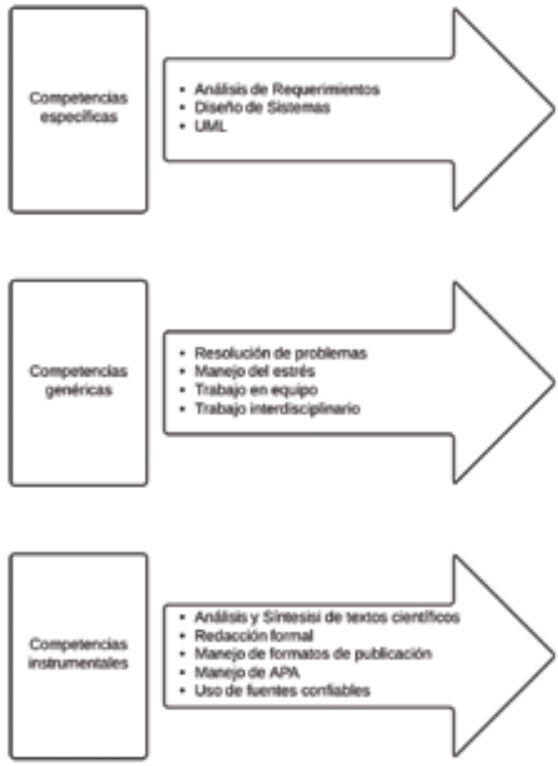

(B)

Figura 1. Competencias a desarrollar en los estudiantes a través de los proyectos (a) Concurso Nacional de Robótica del Sureste y (b) Ponencia en un congreso del área 
En la Figura 1 se pueden observar las competencias a desarrollar con cada una de los proyectos. Para el concurso de robótica se consideraron únicamente competencias específicas y genéricas ya que el énfasis estaría en el trabajo multidisciplinario. Mientras que para la ponencia se consideraron además competencias instrumentales relacionadas con la redacción de textos científicos. Cabe destacar que las actividades se planearon para las 480 horas que dura tradicionalmente un proyecto de servicio social, a una razón de 4 horas diarias durante el semestre Enero - Julio 2013.

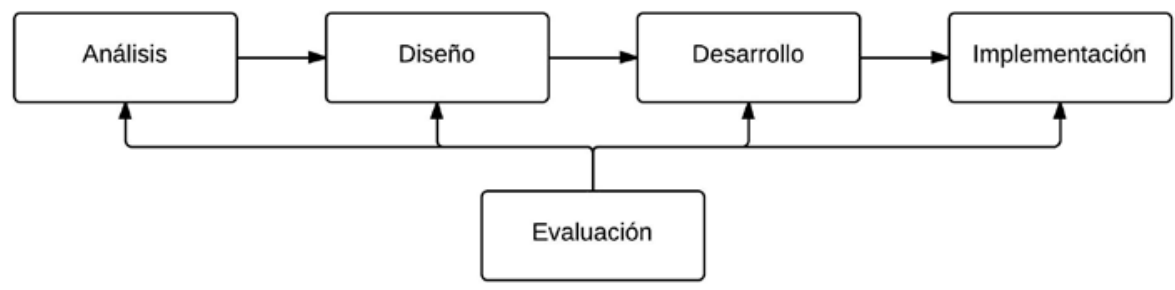

Figura 2. Fases del modelo ADDIE

\section{Actividades}

Los actividades se organizaron de acuerdo con el modelo ADDIE (Yukavetsky, 2003), que se eligió para el diseño de las mismas y se comentan de acuerdo con el análisis que se realizó en cada uno de los momentos de la experiencia. En la Figura 2 se pueden observar las fases y las relaciones del modelo.

\section{Análisis}

La principal meta que se estableció fue desarrollar habilidades complementarias a la formación profesional, llevando a cabo actividades de investigación. Para esto se propuso a los estudiantes el participar en un congreso de talla nacional con una propuesta formal redactada siguiendo el formato de presentación establecido, así como el concurso en el evento de robótica en la categoría de Legos.

Los estudiantes que fueron invitados a participar se encontraban terminando el $5^{\circ}$ semestre de las carreras de Ingeniería en Sistemas Computacionales (ISC) y Electromecánica (IEM) que se caracterizan por su participación y responsabilidad. Debido a lo temprano de su formación, se determinó trabajar con habilidades básicas de su perfil profesional hasta el momento en el que se encontraban. De esta manera se determinó que los estudiantes de ISC diseñaran funcionalmente un sistema de gestión del conocimiento y el estudiante de IEM el diseño e implementación de un cargador de batería para los equipos de Lego recién adquiridos por la escuela. 


\section{Diseño}

Se establecieron las actividades a desarrollar implicando de manera transversal la habilidad de autogestión por parte de los estudiantes y motivando el sentido de la responsabilidad y la actitud al logro, por lo que se enfocó al trabajo autónomo y revisando de manera paulatina y sistemática los avances de cada uno de los proyectos, considerando que los estudiantes marcarían el ritmo de avance de sus actividades planteando un calendario de entrega.

Se consensuaron las estrategias de trabajo y se establecieron períodos en los que los estudiantes recibirían formación formal relacionada con redacción y formatos de publicación, así como períodos en los que se trabajaría con la identificación y ajuste de los robots a utilizar durante el concurso.

\section{Desarrollo}

A modo de ejemplo en la Tabla 1 se puede observar la programación de las actividades establecidas por el grupo de ISC, en ella consta la fecha en la que se entregaría el producto de diseño (requerimientos o diagramas) y la entrega del documento a presentarse en el congreso. En cada una de las entregas los estudiantes consideraron que en la semana posterior el profesor haría una revisión de lo entregado por lo que deberían de realizar correcciones a la par de avanzar con los diagramas que seguían en el calendario.

Tabla 1. Esquema de trabajo propuesto por los estudiantes de Ingeniería en Sistemas para el desarrollo de las actividades de su proyecto

\begin{tabular}{ll}
\hline \multicolumn{1}{c}{ Actividad } & \multicolumn{1}{c}{ Revisión } \\
\hline Requerimientos & 28-feb-2013 \\
\hline Diagrama E/R & 07-mar-2013 \\
\hline Diagrama de casos de uso & 14-mar-2013 \\
\hline Diagrama de secuencia & 21-mar-2013 \\
\hline Agregar otros puntos & 28-mar-2013 \\
\hline Estructurar y dar formato al doc. & 4-abril-2013 \\
\hline
\end{tabular}

Si bien las actividades del Servicio Social deberían de abarcar el semestre lectivo 2013 - A (enero - julio), debieron ajustarse al calendario de actividades del congreso, ya que éste tendría lugar en el mes de Abril, y las propuestas iniciales se enviarían con fecha 28 de Febrero y las propuestas en extenso a más tardar el día 8 de Abril. Con base en estas limitantes, se estableció en conjunto con los estudiantes que los 
ISC modelarían la aplicación mientras que el IEM llegaría a la parte experimental produciendo un primer prototipo del cargador de batería en cuestión.

\section{Implementación}

Durante el tiempo que se designó a las actividades, los estudiantes realizaron un levantamiento de los equipos Legos disponibles en la institución, probaron su correcto funcionamiento y sintetizaron la información disponible sobre su manejo como parte de su estudio, de esta manera conocían no sólo técnicamente con lo que se contaba sino la forma de organizar las partes más eficientemente. Realizaron las pruebas a las que han sido sometidos los estudiantes participantes en ediciones previas del concurso y realizaron mediciones relacionadas con el armado de los robots para la competencia.

En cuanto a sus actividades relacionadas con los proyectos, los estudiantes llevaron a cabo las actividades propias del trabajo a presentarse y en la misma medida se fueron documentando los resultados en el formato del congreso y se revisaron aspectos de redacción y uso de citas y elaboración de referencias, así como algunos recomendaciones del manejo automático de esos elementos en un procesador de texto para que la revisión fuera más dinámica.

Como resultado de los trabajos, ambas propuestas fueron aceptadas para su presentación en el Congreso Nacional de Ingeniería Eléctrica y Electrónica del Mayab. El formato de presentación fue en carteles que describían los puntos más interesantes de cada proyecto, y los estudiantes fueron requeridos para una magna presentación conjunta.

Como parte del registro anecdótico, los estudiantes se retrasaron en algunas entregas debido a sus actividades escolares, por lo que tuvieron que terminar y detallar sus propuestas en un momento en el que no habían actividades escolares y trabajando en línea para llegar a consenso y avanzar en el detalle de los proyectos. El estudiante encargado de diseñar e implementar el cargador de batería debió inclusive asistir a la escuela para hacer uso de las instalaciones y equipo especializado para las mediciones que requería.

Después de realizar las actividades propuestas, los estudiantes participaron con dos ponencias, una denominada Diseño de un Sistema Gestor del Conocimiento aplicados a la Educación Superior y otra denominada Diseño técnico e implementación de un cargador de batería polímero de litio para Lego NTX 9841.

Las actividades de los estudiantes se llevaron a cabo con base en el cronograma aunque con algunos atrasos debido a la calendarización de exámenes y otras actividades extracurriculares en la que participan los estudiantes.

\section{Evaluación de los resultados}


Se determinó llevar a cabo un grupo de enfoque debido a que su uso responde directamente a la necesidad de resolver cuestiones prácticas, suscitadas en el transcurso de la aplicación de herramientas convencionales de investigación social y de mercados (Llopis, 2004). Es una forma de reunir a los participantes de una investigación y obtener de manera directa su perspectiva sobre lo ocurrido durante el desarrollo de las actividades.

Para poder estudiar los resultados del grupo de enfoque se lleva a cabo un proceso de análisis del discurso del estudiante plasmado en sus proposiciones ya que facilita comprender los procesos cognitivos potenciados en el aula y cómo el estudiante construye sus propias representaciones del conocimiento científico (Campos y Gaspar, 2008). Tomando esto en cuenta, se les preguntó su perspectiva acerca de lo conseguido y lo que implicó para ellos el participar en esta experiencia diferente y su impacto en su perfil profesional.

La guía de entrevista se enfocó en cuatro aspectos fundamentales de la experiencia: implicaciones de lo que es el servicio social, la forma en la que se desarrollaron las actividades que se realizaron, los aprendizajes que les dejó la experiencia y una evaluación general del proceso (véase Figura 3).

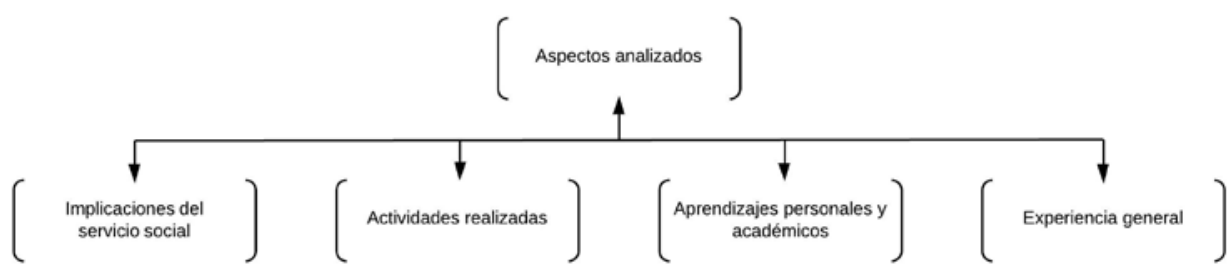

Figura 3. Aspectos de la experiencia que fueron discutidos en el grupo de enfoque

\section{Implicaciones del servicio social}

Uno de los primeros constructos que se trabajaron con los estudiantes fue el Servicio Social, en el que se les preguntó sobre lo qué es y las actividades que se realizan y si consideran que consiguieron cubrir con los aspectos que se trabajan en él.

Los estudiantes reconocen que el principal objetivo del servicio social es el de retribuir a la sociedad algo de lo que ha aportado a su formación profesional, pero en ninguno de los casos se hizo referencia a la aportación a su perfil profesional que debería tener dicho proceso. Considerando este escenario, se necesita reforzar en los estudiantes la idea de que el servicio social no se debe de limitar a buscar una dependencia pública sino a buscar proyectos en los que se pueda desarrollar algún tipo de trabajo relacionado con su área de estudio realizando actividades propias de su perfil. 


\section{Actividades Realizadas}

Las actividades que los estudiantes consideraron como más importantes fueron el desarrollo del proyecto para el concurso en la que trabajaron con Legos para mostrar algunos conceptos básicos de programación.

Los principales retos a los que se enfrentaron fueron el aprendizaje por bloques en el caso de los Legos y el desarrollo escrito de la propuesta del proyecto tanto de forma técnica como de forma general. En este sentido debieron refinar sus habilidades de redacción, programación y diagramación; inclusive uno de los estudiantes mencionó que ahora al leer los trabajos escolares de sus compañeros puede darse cuenta que su habilidad se encuentra más desarrollada y puede indicarles sus errores.

\section{Aprendizajes personales y académicos}

Otro de los puntos focales de su aprendizaje resultó ser el trabajo colaborativo, el llegar a acuerdos, escuchar las ideas de los demás y formas de incorporar nuevas ideas, así como el trabajo multidisciplinario ya que al ser de diferentes carreras al momento de trabajar sobre un mismo proyecto cada quien aportaba su visión a la solución. Aprender a comunicarse mejor de forma sencilla y comprensible para personas de otra formación, ya que al tener una perspectiva diferente sobre la solución hacía que explicaran de forma más detallada sus procesos mentales.

De forma personal todos creen que fue un primer acercamiento al entorno laboral y comprendieron que no siempre colaborarán con personas que entienden sus tecnicismos y hacerlas comprenderlos es parte de un trabajo que garantiza el correcto desarrollo de las actividades.

Asistir al congreso no sólo reforzó su idea del proceso de investigación sino que les permitió ver los avances en diversas áreas y las tecnologías que se están utilizando actualmente y la forma en la que es implementada. Les permitió evaluar su propuesta con las demás que había en el lugar y llegaron a la conclusión de que aunque algunas eran mejores consideraron que otras no presentaban mayor complejidad.

En este sentido el estudiante de IEM consideró que asistir al congreso y participar en la sesión de carteles fortaleció su capacidad de expresarse, ya que como parte de una asignatura tuvo que presentar sus resultados ante sus compañeros y se sintió más confiando y seguro de lo que hacía, resultando en una felicitación por parte de su profesor en turno. 


\section{Evaluación general de la experiencia}

En cuanto a su evaluación general de la experiencia consideraron que el tiempo de desarrollo fue insuficiente, ya que además de hace el servicio debieron realzar actividades escolares (tareas, proyectos, exámenes, etc.) que les robaban tiempo. De la misma manera consideraron que de haber tenido una mejor gestión del tiempo podrían haber enfrentado mejor las responsabilidades.

Los de la carrera de ISC consideraron que les hubiera gustado llegar a la fase de implementación del proyecto y ver en funcionamiento sus ideas, concretar todo lo diseñado y llevar a cabo la documentación correspondiente. Concluyeron que ahora que conocen que el proceso de creación de software puede ser muy complejo aun para una idea muy pequeña, pueden ser más asertivos al momento de planear.

En todos los casos los estudiantes consideraron que pueden idear propuestas innovadoras de desarrollo en los campos disciplinares correspondientes, pero reconocen que sus habilidades aun no les permiten concretar las ideas, al mismo tiempo perciben que son capaces de hacer una investigación que enmarque su proyecto y que pueda ser presentada en algún foro especializado.

En cuanto a su motivación al logro todos coincidieron en que se sienten más seguros de sus conocimientos y de sus habilidades, que reconocen sus capacidades y que se sienten motivados a conseguir más cosas, no limitarse a considerar únicamente actividades escolares sino al desarrollo de aplicaciones más complejas. En definitiva, consideraron la experiencia como positiva y como una actividad que les gustaría repetir de forma constante y permanente.

\section{Conclusiones}

El principal objetivo de esta experiencia fue el ofrecer a los estudiantes del ITS Motul la oportunidad de desarrollar habilidades profesionales y de investigación como parte del Servicio Social que es requisito de titulación de su carrera, debido a que en muchas ocasiones las actividades que realizan no se relacionan con su perfil de formación.

En el caso de la Institución, al estar en proceso de certificar sus programas por organismos externos el promover este tipo de estrategias en los procesos académicos, vinculando a los estudiantes con metodologías formales de investigación permitirá incubar, fortalecer y motivar en los estudiantes la cultura de la investigación, el desarrollo y la innovación, una necesidad específica del subsistema de educación tecnológica que exige de los estudiantes el ser capaces de desarrollar y modificar tecnologías de vanguardia.

En este sentido las actividades fueron cuidadas para que los participantes desarrollaran alguna tarea relacionada con el perfil profesional que se espera de ellos 
y cuyos resultados fueran presentados en un congreso nacional especializado del área.

Al finalizar las actividades se preguntó a los estudiantes sobre su experiencia y lo que les aportó, encontrando que todos coincidían que no sólo fortaleció sus habilidades profesionales sino que además les permitió participar en actividades complementarias que desarrollaron en ellos gusto por la investigación y el desarrollo de proyectos. Coincidieron que aunque han desarrollado proyectos a lo largo de su formación el proceso de formalización de los resultados en términos de un documento les resultó especialmente difícil porque requirió de un lenguaje profesional, pero que al final lo aprendido les redituó en una mayor seguridad al momento de presentarse en público y al momento de redactar un informe son capaces de identificar los errores más comunes en los trabajos. 


\section{Referencias}

Campos, M. y Gaspar S. (2008). Condiciones fundamentales de la construcción de conocimiento, en IX Seminario Internacional de Epistemología, Cognición y Enseñanza de las Ciencias. México: IISUE, UNAM.

Cárdenas, C. (noviembre, 2011). Formación para la investigación: Puntos críticos. Documento presentado en el XI Congreso Nacional de investigación Educativa, D.F., México.

Dirección General de Educación Superior Tecnológica. (2013). Servicio Social. Recuperado el 1 de julio de 2013, de http://www.snit.mx/vinculacion/serviciosocial-dp1

Llopis, R. (2004). El grupo de discusión: manual de aplicación a la investigación social, comercial y comunicativa. Valencia: Editorial ESIC.

Loera, M., \& Carrillo, L. (4 de Octubre de 2004). Con pocas patentes las universidades más importantes de México. La Gaceta, pág. 11.

Monroy, A. (noviembre, 2007). Las patentes y la transferencia tecnológica en las Universidades. Documento presentado en el IX Congreso Nacional de investigación Educativa, Mérida, México.

Olivares, E. (13 de Marzo de 2007). Las universidades públicas producen pocas patentes. La Jornada, pág. 55.

Rivera, E. (noviembre, 2011). Habilidades en el aprendizaje de la Investigación de Estudiantes Universitarios. Documento presentado en el XI Congreso Nacional de investigación Educativa, D.F., México.

Yukavetsky, G. (2003). La elaboración de un módulo instruccional. Recuperado el 23 de agosto de 2005, de Centro de Competencias de la Comunicación de la Universidad de Puerto Rico en Humacao: http://www.ccc.uprh.edu/download/ modulos/CCC_LEDUMI.pdf 
